\title{
Leo Strauss et la Chine : une rencontre autour de l' ethos classique
}

Leo Strauss and China: Discussing the Issue of a Classical Ethos

施特勞斯與中國: 古典心性的相逢

\section{Xiaofeng Liu}

Traducteur : Joël Thoraval

\section{(2) OpenEdition}

\section{Journals}

Édition électronique

URL : http://journals.openedition.org/extremeorient/144

DOI : 10.4000/extremeorient.144

ISSN : 2108-7105

Éditeur

Presses universitaires de Vincennes

\section{Édition imprimée}

Date de publication : 1 octobre 2009

Pagination : 141-154

ISBN : 978-2-84292-237-5

ISSN : 0754-5010

Référence électronique

Xiaofeng Liu, «Leo Strauss et la Chine : une rencontre autour de l'ethos classique », Extrême-Orient Extrême-Occident [En ligne], 31 | 2009, mis en ligne le 01 octobre 2012, consulté le 01 mai 2019. URL http://journals.openedition.org/extremeorient/144; DOI : 10.4000/extremeorient.144 


\title{
Leo Strauss et la Chine : une rencontre autour de l'ethos classique
}

\author{
Liu Xiaofeng \\ Traduction du chinois et notes de Joël Thoraval
}

Dans les années 1980, le monde intellectuel chinois a de nouveau connu une importation à grande échelle de la pensée académique occidentale. Si l'on prend comme référence l'année 1898, au moment où Yan Fu publie une version chinoise de Evolution and Ethics de Thomas Huxley et met la main à sa traduction de The Study of Sociology de Herbert Spencer, on peut considérer qu'il s'agit de la troisième importation massive de ce genre par les intellectuels chinois, importation qui se poursuit de nos jours. De son côté, la période qui s'étend du mouvement du 4 mai 1919 à 1966 peut être vue comme la deuxième étape de ce processus : son résultat le plus remarquable consiste dans la traduction complète des œuvres de Marx, Engels, Lénine et Staline ainsi que dans les versions chinoises des classiques occidentaux, sélectionnés selon la perspective du marxisme. S'il est vrai que le marxisme constitue la forme radicale du mouvement de pensée des Lumières, la traduction en chinois de ces quatre auteurs communistes, qui marque l'aboutissement du processus ainsi ouvert par le 4 Mai, atteste du degré d'institutionnalisation atteint dans l'acceptation de la pensée des Lumières par les intellectuels chinois.

Pourtant, il n'est pas difficile, rétrospectivement, de voir combien chaque introduction depuis l'Occident d' «ouvrages de politique et de droit» a suscité des polémiques dans le monde académique chinois. Autrement dit, les lettrés ou intellectuels chinois ont toujours importé la pensée occidentale de manière sélective - contrairement par exemple au Japon, où cette importation s'est faite de manière plus systématique en suivant chaque fois la mode en vigueur en Occident. Yan $\mathrm{Fu}$, véritable pionnier en la matière, a ainsi critiqué le choix de Rousseau par Liang Qichao, comme si on pouvait faire l'économie d'une telle pensée. Il est clair qu'à l'origine de cette situation se trouve le fait que l'adoption par les lettrés chinois des ouvrages occidentaux de «politique et de droit» a toujours été fonction de la vision qu'ils se faisaient eux-mêmes de la constitution d'une nouvelle Chine. 
Parmi les diverses catégories d' «ouvrages de politique et de droit» que les intellectuels chinois ont importées de l'Occident moderne, la plus importante et la plus influente politiquement est sans conteste le marxisme, même s'il s'est toujours trouvé des lettrés pour s'y opposer (tels les néo-confucéens contemporains). C'est cette association réussie entre un nouveau type d'élite intellectuelle et une pensée des Lumières devenue radicale qui a conduit à un premier remodelage de la nouvelle Chine. Il n'est donc pas difficile de comprendre pourquoi ce sont les écrits de Marx, Engels, Lénine et Staline qui ont constitué, dans le domaine de la politique et du droit, les premières «œuvrescomplètes» traduites en chinois.

La troisième étape d'assimilation de telles œuvres, entamée dans les années 1980, n'a pas manqué de déclencher des polémiques, et cela n'est pas non plus surprenant. Ce qui l'est, c'est plutôt que ces polémiques aient commencé si tard. Je me souviens avoir continûment participé à cette entreprise d'importation et avoir introduit auprès du public chinois de nombreuses théories occidentales, sans pour autant que cela suscite de controverses. Certes, on a pu à l'occasion s'étonner ou critiquer le fait que je m'occupe de présenter la théologie chrétienne, mais sans intention de créer des polémiques. Ce n'est qu'après l'année 2000 qu'ayant successivement introduit la «théologie politique» de Carl Schmitt et la «philosophie politique» de Leo Strauss, j'ai suscité dans le monde académique chinois une attention croissante, qui a ensuite donné lieu à controverses. Ce n'est pas cette réaction qui m'a surpris : ce qui m'a étonné, c'est plutôt que les savants occidentaux, eux aussi, aient prêté une telle attention à l'intérêt que nous portions à Schmitt, et en particulier à sa «théologie politique». Auparavant, personne ne m'avait posé la question de savoir pourquoi je présentais des textes de Heidegger, de Karl Barth ou encore de Max Weber. Or, aujourd'hui, des amis du monde académique américain ou européen ne cessent de me demander: pourquoi diable vous intéresser à Strauss? Naguère, seuls les sinologues ou les spécialistes occidentaux des questions chinoises prêtaient attention aux développements du monde intellectuel chinois. L'écrasante majorité des savants occidentaux ne s'y intéressait pas - alors qu'à l'inverse, on s'intéressait largement, en Chine, à la situation intellectuelle en Occident. Aujourd'hui, ceux qui nous demandent les raisons de notre attirance pour Strauss ne sont plus seulement les sinologues ou les spécialistes des affaires chinoises: l'introduction de Strauss en Chine intéresse dorénavant les intellectuels occidentaux dont les préoccupations ne sont pas seulement tournées vers la Chine mais vers eux-mêmes (l'Europe ou les États-Unis).

Pourquoi? Qui sont ces intellectuels euro-américains et comment expliquer qu'ils se sentent à ce point concernés par notre intérêt pour Strauss? 
Il convient de revenir d'abord à la question qu'ils nous posent: pourquoi Leo Strauss?

Qu'on me permette de commencer par une référence au marxisme. Le marxisme n'a pas disparu du monde intellectuel occidental, les diverses variétés de «nouvelles gauches» sont les fruits du même arbre. Au moins aux États-Unis, en Angleterre, en Allemagne et en France, il existe encore un marché pour le marxisme chez les intellectuels et dans les universités. De fait, personne jusqu'à présent n'est venu s'étonner de notre assimilation de cette doctrine. En revanche, on ne peut dire que chez eux la pensée de Strauss rencontre une telle faveur, alors que c'est de toute évidence le cas aujourd'hui en Chine, même si, il est vrai, un certain nombre d'intellectuels critiquent chez nous cet intérêt, voire s'y opposent violemment. En d'autres termes, la pensée de Strauss que nous souhaitons nous approprier n'est pas en faveur dans le monde intellectuel occidental, lequel préfère Rawls, Giddens, Habermas ou Derrida, et nul ne s'étonnera si nous nous intéressons aussi à ces auteurs, pas plus que ce dernier choix ne soulèvera d'inquiétudes chez les intellectuels chinois. Là encore, pourquoi?

La position intellectuelle de Strauss est connue sous le nom de «philosophie politique». Mais le marxisme n'est-il pas aussi une «philosophie politique»? Qui plus est, non seulement il a acquis une position évidente dans notre monde intellectuel, mais il est au fondement politico-juridique de la Chine nouvelle. Si l'on veut comprendre pourquoi Strauss est si important pour la Chine, il faut prêter attention au fait que la "philosophie politique» dont il se réclame est «classique» (elle a, pour être plus précis, une dimension «platonicienne»).

Aujourd'hui, lorsqu'on parle de philosophie politique, on désigne d'abord une «branche» au sein de la philosophie, au même titre que l'éthique, la religion, l'esthétique ou la théorie sociale. Le fait que, dans l'université d'aujourd'hui, le département de sciences politiques soit distinct du département d'économie ou de sociologie est le résultat ou le reflet d'une telle division du savoir. Il s'agit, comme on le sait, d'une catégorisation qui est le produit de la modernité occidentale: dans les temps plus anciens, en Occident comme en Chine, on ne connaissait pas une telle répartition des branches du savoir. Or, dans le monde scientifique chinois des années 1930, il s'est trouvé des gens pour parler, par exemple, de la «philosophie (ou de la pensée) politique de Confucius», comme si on pouvait trouver chez Confucius ou dans le confucianisme une pensée éthique, une pensée économique, etc On voit ainsi combien le savoir occidental de type moderne a compartimenté le savoir classique chinois, tout autant qu'il l'a fait pour le savoir classique occidental.

Le marxisme est évidemment une «philosophie politique» au sens de cette catégorisation disciplinaire. À strictement parler, il fait partie des «philosophies politiques» qu'on devrait appeler «discours politique» ou «discours en 
- isme», dont relèvent tout autant le libéralisme, le conservatisme, la «nouvelle nouvelle gauche», le structuralisme, le féminisme, le post-colonialisme, etc. Il suffit de feuilleter un manuel actuel de philosophie politique pour tomber sur des «-ismes» de ce genre. Il est clair que ces «-ismes» sont les uns après les autres devenus le savoir en vigueur dans les deux mondes intellectuels, occidental puis chinois.

Les diverses «philosophies politiques» de ce genre ne sont pas des disciplines scientifiques, elles ne se répartissent pas selon les principes de la science moderne: du libéralisme au post-modernisme, ces discours peuvent se manifester dans des domaines scientifiques aussi divers que l'éthique, la science des religions, l'esthétique ou la théorie sociale. Aussi semblent-ils présenter une ressemblance formelle avec la philosophie politique classique. Mais en réalité, si l'on adopte la perspective classique, ils ne sont que des «discours en - isme», plutôt qu'une «philosophie politique». En effet, le mot occidental de «philosophie» a d'abord le sens d'une réflexivité approfondie même dans le cas où cette réflexivité porte sur le «politique» de la «philosophie politique»: elle est d'abord philosophie avant d'être un discours sur la pratique politique. Les divers «discours en - isme», quand bien même ils présupposeraient un arrièrefond philosophique très élaboré, sont d'abord des discours relatifs à la pratique politique et ne sont jamais dépourvus d'un fort caractère agonistique. Devant la prolifération de telles «philosophies politiques», je ne peux m'empêcher de penser aux propos de Nietzsche dans la préface d'Aurore:

Avec des intentions aussi exaltées, Kant était le véritable fils de son siècle qui peut être appelé, plus que tout autre, le siècle de l'exaltation aveugle: comme il l'est demeuré encore, et cela est heureux, par rapport au côté le plus précieux de son siècle (par exemple avec ce bon sensualisme qu'il introduisit dans sa théorie de la connaissance). Lui aussi avait été mordu par cette tarentule morale qu'était Rousseau, lui aussi sentait peser sur son âme le fanatisme moral, dont un autre disciple de Rousseau se croyait et se proclamait l'exécuteur, je veux dire Robespierre, qui voulait «fonder sur la terre l'empire de la sagesse, de la justice et de la vertu»'.

Kant n'est-il pas connu pour la profondeur de sa pensée réflexive? Pourtant, aux yeux de Nietzsche, il est «le véritable fils de son siècle, le siècle de l'exaltation aveugle», c'est-à-dire le XVIII ${ }^{e}$ siècle ou siècle des Lumières. Selon lui, il existe une continuité dans l'esprit de Rousseau, de Kant et de Robespierre. Les «discours en -isme» qui se succèdent depuis le XIX ${ }^{e}$ siècle ne sont pas autre chose que le prolongement de cet esprit - même lorsque certains de ces discours dirigent

1. Nietzsche, Euvres (traduction d'Henri Albert), Paris, Robert Laffont, «Bouquins», 1993, vol.1., p. 969 (traduction légèrement modifiée pour Schwärmerei). N.d.T. 
l'esprit de critique radicale des Lumières contre une version plus ancienne de ces Lumières (comme dans le cas de Derrida).

L'impératif «classique» qui caractérise la philosophie politique promue par Strauss n'est pas autre chose que le rejet fondamental de cette modernité enthousiaste et aveugle (qu'elle soit d'inspiration conservatrice, radicale ou libérale). La première raison pour laquelle nous nous intéressons à Strauss en Chine est qu'il nous permet de nous défaire d'un siècle de quête continue des différents «-ismes», enthousiastes et aveugles, venus de l'Occident.

Mais la «philosophie politique» de Strauss n'est-elle pas aussi un de ces discours en «-isme»?

En aucun cas! Il est impossible d' appeler «straussisme» la «philosophie politique» de Strauss, car son impératif «classique» est littéralement dirigé contre l'ensemble des «discours en -isme» de la modernité. En tout état de cause, à considérer son essence, la «philosophie politique» défendue par Strauss exige, premièrement, une révision complète de la philosophie politique issue des temps modernes (de Rousseau, Kant ou Robespierre jusqu'à, de nos jours, Derrida) et pour ce faire, elle exige, deuxièmement, que l'on comprenne les classiques à la lumière de l'auto-compréhension des Anciens eux-mêmes et que l'on interprète les discours politiques modernes selon la perspective des classiques. Si la position intellectuelle de Strauss n'est pas en vogue dans le monde académique occidental, c'est précisément parce que les divers « discours en -isme» modernes détiennent une suprématie absolue dans les universités occidentales - aussi serait-il, du reste, quelque peu risible que des savants européens ou américains ironisent sur la domination absolue du marxisme dans les universités chinoises. À l'inverse, les positions de Strauss provoquent un certain malaise chez des intellectuels chinois justement parce que les divers « discours en - isme » qu' ils poursuivent ardemment (qu'ils soient libéraux ou conservateurs) se trouvent ainsi soumis à une interrogation et à un défi radical.

Au fond, qu'il s'agisse de l'Occident ou de la Chine, les fondements devenus institutionnels du monde intellectuel et universitaire trouvent leurs origines dans les Lumières du XVIII ${ }^{\mathrm{e}}$ siècle européen. La différence, c'est qu'en Occident les différents «discours en -isme» issus des Lumières ont déjà été complètement institutionnalisés, alors qu'en Chine on est toujours dans un processus visant à réaliser cette institutionnalisation. En conséquence, la signification effective de la philosophie politique classique de Strauss est encore plus grande en Chine qu'en Occident.

La carrière universitaire de Strauss ne ressemble guère à celle de professeurs comme Derrida, dispensant leur enseignement aux quatre coins du monde. Dès lors, pourquoi faut-il qu'à peine présentées par nous, les conceptions de Strauss suscitent le malaise et même l'inquiétude chez certains intellectuels et 
professeurs d'université ? C'est parce que nos professeurs d'université ont été formés à l'école d'une pensée à la Robespierre, visant «àfonder sur la terre l'empire de la sagesse, de la justice et de la vertu»... C'est ce qu'on peut appeler le grand «dessein moderne» et, pour réaliser ce dessein, il faut fonder sur cette terre un système éducatif qui puisse le servir. Aujourd'hui, cette institution s'est complètement développée en Europe et en Amérique, tandis qu'en Chine le système éducatif s'est efforcé depuis quatre-vingt dix ans de suivre cette voie à marche forcée.

Comment établir «sur la terre» un système éducatif complètement modernisé ? On le voit clairement si l'on considère l'anthropologie, une discipline qui exerce véritablement dans l'université d'aujourd'hui une fonction directrice: de fait, les préceptes de l'anthropologie sont déjà devenus les principes fondamentaux de l'éducation supérieure. Souvenons-nous que l'anthropologie, bien qu'elle ait atteint la Chine à l'époque républicaine au travers des œuvres de Malinowski, britannique d'origine polonaise (1884-1942), n'est devenue en Chine une discipline en vogue que dans les années 1980, époque où l'anthropologie structurale, portée par la réputation dont jouissait alors en Europe le structuralisme, atteignait notre pays. Il est connu que le mouvement de révolte étudiante de 1968 en France n'est pas sans lien avec l'essor du structuralisme. Ce mouvement entendait éliminer les grands livres classiques des amphithéâtres, au nom de l'égalité des formes de vie - car l'anthropologie structurale est sans doute moins un «mouvement intellectuel» qu'un «mouvement politique». Il n'est pas difficile de voir que les discours politiques qui suivent le structuralisme (les discours post-structuralistes comme ceux de Foucault ou Derrida) sont aujourd'hui toujours en vogue non seulement en Europe mais aussi ici, en Chine.

L'anthropologie n'étudie pas seulement les modes de vie des descendants des «sociétés primitives » mais également la civilisation classique. Parmi les maîtres de l'anthropologie structurale française, on compte Claude Lévi-Strauss mais aussi Jean-Pierre Vernant. Les spécialistes français de l'Antiquité sont nombreux. Or, nous n'avons pratiquement pas traduit leurs livres. En revanche, on dispose de traductions d'au moins trois ouvrages de Vernant. De toute évidence, la popularité de Vernant dans le monde intellectuel chinois n'est pas due au fait qu'il est un spécialiste de la Grèce classique mais au fait qu'il est un représentant de l'anthropologie structurale d'inspiration marxiste. L'anthropologie religieuse de la Grèce ancienne de Vernant et l'anthropologie religieuse des sociétés primitives de Lévi-Strauss sont comme les deux lames d'une même épée pour couper le lien avec la tradition classique et transformer les principes de l'éducation traditionnelle, faisant ainsi d'une pierre deux coups. Comme le dit Vernant luimême, l'objet de recherche de Lévi-Strauss est passé de «la religion des peuples 
non civilisés » à une «étude comparée des religions des peuples sans écriture». Sa méthode consiste à établir des analogies structurales entre des discours et des phénomènes sociaux relevant de sociétés de types différents, afin d'établir un «phénomène social total», pour en induire une théorie à valeur universelle. Vernant lui-même n'a pas fait autre chose qu'accomplir ce même travail au sujet de la religion d'une Grèce antique parvenue à la civilisation et développer ainsi l'étude comparatiste de la religion d'un peuple possédant l'écriture. Ce faisant, il faisait pénétrer l'anthropologie dans les études classiques, ce cœur de l'enseignement des humanités en Occident ${ }^{2}$.

L'association de l'anthropologie et des études classiques n'est pourtant pas une création originale qui serait due à Vernant. Dans ce centre des études classiques qu'est la ville de Bâle en Suisse, en 1861, Johann Jakob Bachofen avait déjà publié son livre, Das Mutterrecht, dans lequel Engels avait vu une "véritable révolution», dans la mesure où il renversait complètement les conceptions sociales traditionnelles et restées dominantes, fondées sur le «préjugé patriarcal» ${ }^{3}$. Si l'on voit dans l'éducation de type classique l'une des manifestations directes d'un «préjugé patriarcal» traditionnel, alors, renverser ce préjugé revient à mettre à bas l'éducation de type classique. Dans ce livre, Bachofen a ouvert la voie à l'étude des religions primitives, de la mythologie et de la différence des genres, et son influence est évidente dans les sciences humaines de l'université moderne (son développement ultime étant les diverses cultural studies d'aujourd'hui). Ce n'est pas sans justifications qu'au lendemain du mouvement étudiant européen de 1968, des voix se sont élevées au sein du monde académique pour mettre en valeur la dette ainsi contractée envers Bachofen.

D'où vient l'intuition de Bachofen dans sa doctrine sur le «droit maternel»? Elle vient des théories sur la mythologie du romantisme allemand, ou plus exactement de la compréhension qu' avaient les penseurs romantiques des récits mythologiques de la Grèce. De plus, on peut faire remonter les origines des théories des romantiques sur la mythologie aux conceptions des penseurs français des Lumières relatives à l'Antiquité et aux coutumes populaires. De sorte qu'il est tout à fait possible, si l'on considère la relation interne que Nietzsche reconnaissait dans l'esprit de Rousseau, Kant et Robespierre, de la voir se prolonger directement dans les divers «discours en -isme» du monde intellectuel occidental d'aujourd'hui.

2. Cette position anti-anthropologique est développée (notamment au sujet de J.-P. Vernant et R. Girard) dans «Renleixue de yuwang yu gudian» (Le désir anthropologique et les classiques), in Jian jin han zhi («Choisissant les branches devenues froides »), Huaxia chubanshe, 2007, p. 146-159. N.d.T.

3. Bachofen Johann Jakob, Das Mutterrecht [1861], Francfort, Suhrkamp, 1997. 
Le fait que les principes de l'anthropologie occupent une position dominante dans le système éducatif contemporain implique que l'enseignement supérieur ne doive plus apprendre aux étudiants à porter leur regard vers l'âme des grands auteurs apparus dans l'histoire, mais sur leurs propres désirs et imaginations. Les étudiants n'ont plus besoin de lire les livres classiques consacrés: il suffit qu'ils s'interrogent sur leurs aspirations, leurs souvenirs ou leurs fantasmes pour se former par eux-mêmes à devenir des hommes «moraux ».

Quels sont, dès lors, les effets de tels principes éducatifs? Comme ce philologue qu'était Nietzsche l'avait bien vu, il y a déjà un siècle, le fondement moral de la vie humaine finit par s'estomper et disparaître.

Si l'on compare notre façon de vivre à celle de l'humanité pendant des milliers d'années, on constatera que, nous autres, hommes d'aujourd'hui, vivons dans une époque très immorale: le pouvoir des mœurs est affaibli d'une façon surprenante et le sens moral s'est tellement subtilisé et élevé que l'on peut tout aussi bien le considérer comme volatilisé (Aurore, § 9) 4 .

La deuxième raison pour laquelle nous nous approprions Leo Strauss est la suivante. Il nous faut refuser le système éducatif occidental apparu avec la modernité. Pourquoi ? Parce que l'université et le monde académique ont cessé de traiter de la différence entre le vrai et le faux, le juste et l'injuste, le bon et le mauvais, le beau et le laid. Les gens qui ont reçu une éducation supérieure (et en particulier les professeurs) sont inférieurs aux gens ordinaires sur le plan moral. En effet, ils prétendent ne pas avoir eux-mêmes à parler de vertu, au nom de la «neutralité axiologique ${ }^{5} »$.

De quoi devons-nous nous servir pour nous opposer aux principes de l'anthropologie?

De ce point de vue, l'éducation classique que préconise Strauss nous suggère un tournant possible, car elle nous engage à fixer notre regard vers les plus hauts esprits de l'Antiquité, ce qu'on ne peut percevoir qu'au travers des ouvrages classiques que ces temps anciens nous ont légués. Pour traiter du vrai et du faux, du juste et de l'injuste, du bon et du mauvais, du beau et du laid, nous devons nous fonder sur un ensemble de principes moraux, que la civilisation antique, que ce soit en Occident ou en Chine, a déjà très tôt énoncés ${ }^{6}$. L'anthropologie $a$ rejeté ces principes, mais nous devons les chercher à nouveau si nous souhaitons recouvrer le sentiment moral digne d'un homme de culture (xueren).

\footnotetext{
4. Nietzsche, CEuvres (traduction d'Henri Albert), op. cit., vol. 1, p. 975.

5. Voir Leo Strauss, Thoughts on Machiavelli, Chicago, The University of Chicago Press, 1958, p. 9-14.

6. Voir Nietzsche, Antéchrist, in Euvres, op. cit., vol. 2, § 57-59, p. 1094-1100.
} 
Pourquoi les positions intellectuelles de Strauss ont-elles assez vite suscité un tel écho dans l'université et le monde académique chinois?

Mon interprétation est la suivante: notre université et notre monde académique en sont encore à essayer de suivre le plus vite possible la voie tracée par leurs équivalents occidentaux, alors même qu'ils ne sont pas encore parvenus à constituer pleinement une université et un monde académique de type occidental. Si notre monde universitaire et intellectuel était déjà, sur le plan institutionnel, comme en France ou en Allemagne, complètement transformé par le modèle américain, les conceptions de Strauss n'auraient aucune chance de connaître une telle vogue. À l'inverse, c'est justement parce que nos institutions universitaires et savantes ne sont pas encore parvenues à rejoindre ces modèles occidentaux (ou, pour mieux dire, parce qu'ils ont carrément échoué dans cette tentative), que nous devons profiter de cette situation pour promouvoir au plus vite l'éducation classique.

L'esprit des Lumières devenu radical a effacé en Chine l'horizon de l'ancien «empire céleste» pour lui substituer le nouveau monde universalisé de l'Occident. C'est là l'apport du marxisme à la nouvelle Chine. Mais quiconque connaît l'histoire intellectuelle de la Chine au $\mathrm{xx}^{\mathrm{e}}$ siècle sait parfaitement que le problème rencontré par les marxistes chinois a toujours été celui de la sinisation du marxisme. En dernière analyse, la question qui s'est nécessairement posée à eux a été: qu'en est-il de l'ethos de la Chine? Après que la Chine marxiste est entrée dans l'âge de la globalisation, quel lien vital de filiation peut-elle entretenir avec la Chine traditionnelle ?

Ce lien vital entre Chine nouvelle et Chine traditionnelle est une question qui est restée incontournable pour les intellectuels contemporains chinois - les confucéens parmi eux ayant toujours représenté à ce sujet une force essentielle. Depuis la fin de la dynastie des Qing, la situation à laquelle le monde intellectuel chinois a dû se confronter est celle d'une disruption et d'une fragmentation des «arts de la voie» (daoshu), c'est-à-dire des enseignements traditionnels, après la pénétration en Chine du savoir occidental - qu'il s'agisse de rivaliser pour introduire les versions alors en vogue de ce savoir occidental ou, au contraire, de s'opposer à lui. Ce qui donne à réfléchir, c'est le fait que même les confucéens contemporains qui prétendent préserver la tradition éthique de la Chine se sont, presque sans exception, appuyés sur toutes sortes de théories occidentales modernes. Ils ont réinterprété la tradition de pensée confucéenne de manière critique ou par extrapolation en partant de la philosophie de Kant, ou encore ils ont réinterprété l'esprit du confucianisme en s'appuyant sur la théorie sociologique de Max Weber, produisant ainsi ce qui a été reçu un certain temps comme une «relecture» sophistiquée du confucianisme. Ce n'est pas différent 
des deux siècles où, en utilisant toutes sortes de «théories » modernes, l'Occident a réinterprété sa propre tradition classique.

La troisième raison de notre désir d'introduire Strauss en Chine est la suivante: la «philosophie politique classique» de Strauss nous donne la possibilité de nous arracher à la perspective conventionnelle consistant à évaluer la tradition classique chinoise à l'aune des théories occidentales modernes, et de nous débarrasser de toutes sortes de phénomènes politiques «enthousiastes et aveugles » qui habitent le système d'enseignement des humanités dans l'Occident moderne. Nous pouvons ainsi nous inspirer de la sagesse des Anciens pour mettre en doute et faire obstacle aux «-ismes» modernes, et non, à l'inverse, partir des «-ismes» modernes pour corriger la sagesse des Anciens.

Que ce soit dans les milieux intellectuels chinois ou euro-américain, quiconque exprime son adhésion aux conceptions classiques défendues par Strauss s'expose à une mise en cause radicale: Strauss est un penseur de la droite extrême, adversaire des Lumières et de la politique démocratique. Contre ce genre d'accusation, il n'est ni souhaitable ni possible de se dérober. Je souhaiterais faire deux remarques. D'abord, l'auteur de ce genre de réaction manifeste qu'il se tient lui-même sur les positions de l'idéologie des Lumières. Or, la contestation de Strauss est précisément dirigée contre les Lumières modernes. Une telle réfutation n'est donc pas, à son tour, à réfuter: ce dont il s'agit, c'est de choisir qui est son ami et qui son ennemi. En second lieu, si nous ne commençons pas à apposer sur Strauss une étiquette d' «extrême droite» ou d' «adversaire des Lumières » et que nous prenons un peu de recul pour mieux percevoir sa position, nous pouvons observer ceci : l'effort de Strauss consiste essentiellement à rouvrir la question du caractère moral des Lumières et de la politique démocratique. La légitimité des Lumières, et surtout celle de la démocratie, a atteint le point où l'on ne peut plus la discuter ou la mettre en cause. Mais en tant qu'homme intéressé à la sagesse, Strauss revendique de manière obstinée le droit du philosophe au doute, à la mise en question. Grâce à cette mise en doute, il nous permet de percevoir que la question de savoir si l'on est l'ami ou l'ennemi des Lumières et de la politique démocratique, que la possibilité ultime de ce choix n'est pas à trouver ailleurs que dans la préférence revendiquée par l'individu.

Que 1'on me permette de m'expliquer, grâce à un exemple concret. Dans le livre qu'il consacre à Machiavel, Strauss, à un certain moment, voit dans ce dernier un précurseur de la pensée démocratique moderne. Comment et pourquoi Machiavel peut-il être un tel précurseur? L'interprétation de Strauss est la suivante.

Machiavel tourne le dos à «l'ensemble des auteurs de l'antiquité occidentale», il «s'oppose à l'ensemble de la tradition» et à l'opinion commune (the common opinion). En d'autres termes, l'opinion des auteurs classiques sur 
«la sagesse et la constance de la multitude» (the wisdom and constancy of the multitude») n'est pas autre chose pour lui que celle qui est dictée par le sens commun $^{7}$. On voit ainsi que le point crucial de la dispute entre les anciens et les modernes vient d'une divergence fondamentale sur cette question de «la sagesse et la constance de la multitude». La conception antique se fonde sur l'opinion commune, produit du sens commun. Et la conception moderne? Elle s'appuie sur une certaine philosophie. Car Machiavel pense que la multitude, comparée à cet homme seul qu'est le prince, possède davantage de sagesse et de constance. Il fait de la voix de la multitude «une opinion universelle» $(a$ universal opinion), voire «la voix même de Dieu». Si l'on rapproche les deux expressions précitées, «l'opinion commune» et «une opinion universelle», on voit que cette notion d' «universel» laisse transparaître quelque chose: ce quelque chose est une philosophie. L'universel désigne une conception philosophique, c'est pourquoi elle entretient un rapport antagoniste avec la conception issue du «sens commun». En d'autres termes, le point de vue de Machiavel repose sur une philosophie: l'opposition des Modernes aux Anciens n'est pas autre chose que l'opposition de la philosophie au sens commun. C'est pourquoi Strauss maintient que Machiavel met en question, au nom de la multitude et de l'institution démocratique, la figure du «premier sage» en tant qu'elle incarne le préjugé et le présupposé de ce que doit être un bon régime politique. Il faut ici prêter attention à deux points: $1^{\circ} 1^{\prime}$ idée même de multitude (ou de majorité) fait écho à celle de choix qui caractérise le «régime démocratique » et la démocratie est le régime politique consacrant le règne de la multitude $; 2^{\circ}$ la philosophie classique reflète le préjugé et le présupposé du bon régime (Mesdames et Messieurs, prenez garde: si nous étudions maintenant la philosophie classique, c'est en nous mettant à l'école du préjugé et du présupposé du bon régime! Il en va de même du confucianisme traditionnel de la Chine, malgré l'erreur complète de certains confucéens modernes qui croient trouver la pensée démocratique dans le confucianisme classique). Pourquoi une telle attitude chez Machiavel, telle que la présente Leo Strauss ? Parce qu'il pense que «les aspirations du peuple» (the purpose of the people) sont «plus honnêtes ou plus justes» (honest or just) que «les aspirations des grands» (the purpose of the great). On peut remarquer que la «multitude» dont il était question auparavant est devenue «le peuple», et que le «prince» est devenu «le grand». Est-ce de manière fortuite que Machiavel fait usage de ces mots? Naturellement pas. Dans les deux cas, le changement de termes exprime une réduction car le prince chez les Anciens doit aussi être un «grand». Dans un troisième glissement de vocabulaire, ce qui correspond

7. Il convient de noter que Liu Xiaofeng traduit le terme de «multitude» par le mot duoshuren, dont le sens propre est: «majorité». N.d.T. 
à la «sagesse et la constance» est à présent «l'honnête et le juste»: la qualité d'ordre philosophique a ainsi été réduite à une qualité d'ordre moral ${ }^{8}$.

Mais Strauss poursuit en disant qu'en réalité, Machiavel «n'est nullement attaché au gouvernement par la multitude», car il voit très clairement que «tous les régimes simples » sont «mauvais ». En effet, ils aboutissent soit à l'anarchie soit à l'oligarchie. Or tous les régimes démocratiques sont en définitive des «régimes simples». S'il en est ainsi, pourquoi Machiavel continue-t-il à préconiser le régime démocratique? N'est-ce pas contradictoire ? Comment peut-il à la fois savoir que le régime démocratique est «mauvais» et malgré tout se montrer infidèle à la sagesse classique en soutenant un tel régime? L'interprétation finale de Strauss est que l'explication est à chercher du côté du tempérament et du caractère de l'homme Machiavel : «son inclination naturelle en faveur de la multitude le rendait capable ou le contraignait à ne pas simplement se reconnaître» dans le bon régime de la tradition classique. On pourrait trouver qu'une telle interprétation est trop simple ou carrément triviale. Mais au contraire, ce qu'il faut remarquer, c'est que la réflexion de Strauss n'est pas à la recherche d'un argument particulièrement sophistiqué mais qu'elle préfère énoncer des vérités du sens commun. Comme ce que nous apprend notre expérience quotidienne, il n'y a pas à chercher plus loin les raisons qui font que quelqu'un possédant telle ou telle conviction agit de telle ou telle façon: c'est seulement que son caractère est ainsi, et c'est tout.

8. L'usage que fait Liu Xiaofeng du mot «philosophie» est pris dans ce paragraphe en deux sens différents (ancien et moderne), et peut ainsi prêter à confusion. Cette «certaine philosophie» que l'on peut repérer implicitement chez Machiavel est, selon Leo Strauss, le début de la philosophie politique moderne. Celle-ci reste enfermée dans les limites d'une cité dont l'horizon philosophique a été abaissé, se réduisant désormais à «conduire l'homme vers la société rationnelle, dont le lien et la fin consistent dans l'intérêt privé mais éclairé et dans l'auto-préservation de chacun de ses membres » (Thoughts on Machiavelli, op. cit., p. 296). Ce changement représente une « réduction», indique Liu Xiaofeng, par rapport aux idéaux philosophiques des Anciens. En effet, écrit Strauss, «les classiques comprenaient le phénomène politico-moral à la lumière de la perfection ou des plus hautes vertus de l'homme, la vie du philosophe ou la vie contemplative. La philosophie transcende la cité, et la valeur de la cité dépend en dernière analyse de son ouverture ou de son respect vis-à-vis de la philosophie. Pourtant, la cité ne peut remplir sa fonction sans être à la fois ouverte et fermée à la philosophie: la cité est nécessairement la caverne (de Platon). La cité comprise dans sa fermeture à la philosophie est le demos au sens philosophique, c'est-à-dire la totalité des citoyens qui sont incapables ou non désireux de s'en remettre à la philosophie. Les philosophes et le demos, dans le sens indiqué ici, sont séparés par un gouffre: leurs fins diffèrent radicalement. Ce gouffre ne peut être comblé que par une noble rhétorique » (op. cit., p. 295-296). N.d.T. 
Certains diront qu'utiliser l'enseignement de Strauss pour interpréter les classiques de la Chine revient encore à faire usage, à cette fin, de théories occidentales modernes. Mais cette vision des choses est erronée car Strauss ne met d'aucune manière en avant une théorie qui lui serait propre (du type des théories herméneutiques) pour interpréter les classiques: il entend au contraire lire les ouvrages classiques avec le regard des auteurs classiques. Au cours du $\mathrm{xx}^{\mathrm{e}}$ siècle, il s'est déjà trouvé en Chine des savants pour déplorer que depuis les Tang, les lettrés ne comprennent plus le sens de l'enseignement des anciens et se montrent incapables de parvenir aux hauteurs atteintes par ces derniers, et pour s'efforcer de revenir à la manière originelle de lire les textes. On n'en conclura naturellement pas qu'il s'agit là de s'emparer d'abord d'une théorie moderne pour l'appliquer ensuite à la lecture des livres anciens. De même, notre appropriation de la «philosophie politique classique» défendue par Strauss diffère des théories en vogue introduites en Chine depuis cent ans. Notre entreprise n'est pas une théorie en «-isme» ni une «nouvelle méthodologie», mais une orientation intellectuelle ou, pour mieux dire, elle consiste dans une disposition d'esprit classiciste. Certes, lorsque nous présentons les «conceptions» de Strauss au sujet de la politique et de la philosophie, ces «conceptions» nous viennent bien d'un professeur d'université américain. Mais Strauss est le symbole d'une disposition d'esprit attachée aux classiques et non d'une théorie parmi d'autres (encore moins d'une doctrine politique). Introduire Strauss en Chine, c'est inviter à la rencontre avec une disposition d'esprit fidèle aux classiques. Depuis trois cents ans en Occident et près de cent ans en Chine, cette disposition d'esprit s'est égarée, elle a perdu ce qui constitue son foyer: l'École (xuetang). Aujourd'hui, que ce soit en Occident ou en Chine, cet esprit est à la recherche, au sein de l'université post-moderne, d'un espace qui lui soit propre.

Qui sont en définitive les savants européens et américains qui prêtent attention à l'introduction de Strauss en Chine ? Ce sont, j'en suis sûr, des hommes de culture. Que ce soit en Chine ou en Occident, ce type d'homme représente une minorité au sein d'une université et d'un monde académique entièrement démocratisés. La seule différence est que, la population de la Chine étant plus importante, une minorité de ce genre y est nécessairement plus nombreuse, voilà tout... 
Liu Xiaofeng

\section{Glossaire}

daoshu 道術

duoshuren 多數人

Liang Qichao 梁啟超

xueren 學人

xuetang 學堂

Yan Fu 嚴復 